\title{
Electrochemical immunosensor based on MWCNT-CS as a signal amplification strategy for the detection of capsaicinoids
}

\author{
Fengzhen Yang ${ }^{1,2,3}$, Qingqing Yang ${ }^{1,2,3}$, Jiansen Li $^{1,2,3}$, Qianqian Kong ${ }^{1,2,3}$,Jingcheng Huang ${ }^{1,2,3}$, \\ Rui Xu ${ }^{1,2,3}$, Yaodong Xiang ${ }^{1,2,3}$, Yemin Guo ${ }^{1,2,3, *}$ \\ ${ }^{1}$ School of Agricultural Engineering and Food Science, Shandong University of Technology, No.266 \\ Xincun Xilu, Zibo 255049, China \\ ${ }^{2}$ Shandong Provincial Engineering Research Center of Vegetable Safety and Quality Traceability, \\ No.266 Xincun Xilu, Zibo 255049, China \\ ${ }^{3}$ Zibo City Key Laboratory of Agricultural Product Safety Traceability, No.266 Xincun Xilu, Zibo \\ 255049, China \\ *E-mail: gym@sdut.edu.cn
}

Received: 4 August 2021 / Accepted: 16 September 2021 / Published: 10 October 2021

\begin{abstract}
We constructed an electrochemical immunosensor based on multiwalled carbon nanotube-chitosan (MWCNT-CS) for on-site detection of capsaicinoids, which are label compounds in waste oils. In this work, we fixed capsaicinoids antibodies (Abs) and ovalbumin (OVA) on the surface of the MWCNTCS complex. The good adsorbability and conductivity of MWCNT was utilized to greatly enlarge the current response signal. OVA was embedded in the nonspecific sites of the Abs to enhance the detection sensitivity of the immunosensor. Cyclic voltammetry (CV) and differential voltammetry (DPV) were used to characterize the working principle and current response signals of the immunosensor. The parameters affecting the current response signal, such as the $\mathrm{pH}$ of the buffer solution, incubation time and Abs concentration, were optimized. The linear range of the immunosensor was $2-100 \mu \mathrm{g} / \mathrm{mL}$, and the limit of detection (LOD) was $0.25 \mu \mathrm{g} / \mathrm{mL}$. The aptasensor provides the advantages of high sensitivity, short time consumption and high stability. Overall, the results demonstrated that the developed method could meet the demands of rapid on-site assays for capsaicinoids in waste oil.
\end{abstract}

Keywords: Capsaicinoids; Immunosensor; Multiwalled carbon nanotube

\section{$\underline{\text { FULL TEXT }}$}

(C) 2021 The Authors. Published by ESG (www.electrochemsci.org). This article is an open access article distributed under the terms and conditions of the Creative Commons Attribution license (http://creativecommons.org/licenses/by/4.0/). 